\title{
H-LEACH Protocol with Fault Tolerance in WSN
}

\author{
Ratinder Pal Kaur ${ }^{1}$, Er. Rupinder Kaur ${ }^{2}$ \\ Bhai Gurdas Institute of Engineering \& Technology, Sangrur ${ }^{1}$
}

\begin{abstract}
The wireless sensor network is the type of networks in which sensor nodes sense the network information and pass the sensed information to the base station. Due to small size of the sensor nodes, the battery of the sensor nodes is very limited. In the recent times, various techniques has been proposed which will reduce energy consumption of the wireless sensor networks. In this paper, we evaluate and analyze H-LEACH protocol which is the combination of HEED and LEACH protocol. In this we apply Boltzmann learning technique to select intermediate node between Cluster Head and the Base Station for fault tolerance and to increase the network lifetime. The simulation results show that our method has less delay, energy consumption, less packet loss and high throughput.
\end{abstract}

Keywords: H-LEACH, Wireless Sensor Network.

\section{INTRODUCTION}

Wireless sensor networks are a kind of network system with low energy consumption, self-organization, diverse structure and an extensive connection which is composed of a large number of wireless sensor nodes equipped with sensing devices, and are densely distributed for specific applications. In wireless sensor network collection of nodes organized into a cooperative network. Each node consists of processing capability (one or more microcontrollers, CPUs or DSP chips), may contain multiple types of memory (program, data and flash memories), have a RF transceiver (usually with a single Omni-directional antenna), have a power source (e.g., batteries and solar cells), and accommodate various sensors and actuators. The nodes communicate wirelessly and often self-organize after being deployed in an ad hoc fashion. The purpose is to work together to sense, collect and process information in the network coverage area, and send it to the observer. It is widely used in military defence, industrial and agricultural control, urban management, medical, biological, environmental monitoring, disaster relief, anti-terrorism, risk area remote control and many other areas, and it has very broad application prospects.

\subsection{Architecture of a WSN}

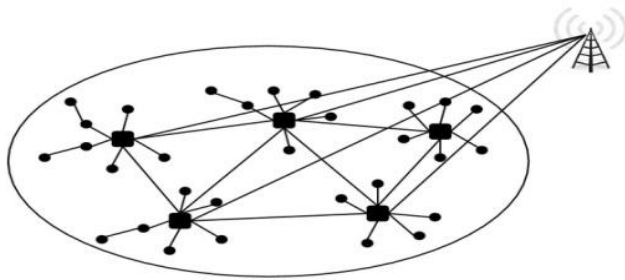

- Sensor node
Cluster head node
A Base station
- SensorNode: Sensor node is the important component of WSN because of its multiple role features. It senses data, stores data, routes data and processes data.

- Clusters: Clusters are small manageable units which simplify tasks such a communication.

- Cluster heads: Cluster heads are the leader who organizes cluster activities. It collects data from several sensor nodes and then aggregates those data and also organizes the schedule of a cluster for communication with BS.

- Base Station: Base station is a central component which collects data from several nodes distributed at different locations. It acts as an intermediate between the network and end-user.

\subsection{Some Issues of Wireless Sensor Networks}

Heterogeneity: There are two types of WSN networks, homogeneous and heterogeneous.

Topology: Topology maintenance of the way the nodes are deployed and how network evolves over time is very challenging. The network is more vulnerable to tampering and failure

Power Consumption: The nodes in the network are battery operated and hence possess finite amount of energy.

Transmission Medium: The communication medium for WSN is Wireless and hence prone to many attacks.

Fault Tolerance: The WSN is vulnerable to node failure and hence the network should function normally even in presence node failures.

Scalability: With the increase in the number of nodes the network should still be functioning appropriately.

Operating Environment: The WSN can be deployed on different applications that needs to be approached in different ways.

Clustering: For effective management of WSN nodes can be grouped into non overlapping groups and each of these 
groups are led by a Cluster Head that manages the activities and scheduling of the nodes.

Security: Due to their communication media, environment and nodes vulnerability WSN are more susceptible to security breaches called attacks. Security attacks can be Active or Passive.

\subsection{Energy Consumption of Sensor Node}

A critical constraint on sensors networks is that sensor nodes employ batteries and deployed them in unattended and in large numbers, so that it will be difficult to change or recharge batteries in the sensors. Therefore, all systems, processes and communication protocols for sensors and sensor networks must minimize power consumption. The existing research on energy consumption of sensors is usually based on either theoretical models or computer simulations. One widely cited model of energy consumption by Heinzelman et.al [13] has been used extensively as a guide for simulations and the design of low power consumption communication protocols The sensor nodes operate in the three modes of sensing, computing and communications, and all of which consume energy. Of the three modes, maximum energy is expended for the communications process. Communication energy contributes to data forwarding and it is determined with the transmission range that increases with the signal propagation in an exponential way. The energy consumption model includes the five states: Acquisition, Transmission, Reception, Listen and Sleep []. Since the sensor nodes can be in any of three main operations of sensing, computations and communications, each of them could be in different states depending on the component nature. Accordingly different levels of energy are expended in each of them.

i. Acquisition: The acquisition state includes sensing, A/D conversion, pre-processing and eventually storage of these data.

ii. Transmission: The transmission state includes processing, packet forming, encoding, framing, queuing and base band adapting to RF circuits.

iii. Reception: This state is responsible for low noise amplification, down converter oscillator, filtering, detection, decoding, error detection, address checking and random reception.

iv. Listen: The listen state is similar to reception and involves the processes of low noise amplification, down convertor oscillator, filtering and terminates at detection.

v. Sleep: The sleep state expends least energy as compared to the other states.

\section{LITERATURE REVIEW}

Abdul Razaque et al. ${ }^{[1]}(\mathbf{2 0 1 6})$, They proposed a new protocol called H-LEACH which is the combination of HEED and LEACH protocol. H-LEACH is more efficient than existing LEACH protocol. It comes over the node energy issues which is the main disadvantage of LEACH protocol. A new formula is proposed in this paper to find the threshold value by using the average energy of the node. The energy consumed by the node for transmitting and receiving data is reduced in every round to keep track of the alive nodes in every round. Node is declared dead when its energy falls below the minimum energy required to transmit energy.

BlessyVarghese $^{[2]}(\mathbf{2 0 1 6})$, He discuss the various protocols in Cluster Head Selection used in Wireless Sensor Networks. Protocols in Wireless Sensor Networks must be energy efficient, to become prolong network lifetime. Wireless Sensor Network is a wide area, this paper has included only few routing protocols like LEACH (Low Energy Adaptive Clustering Hierarchy), BCDCP (BaseStation Controlled Dynamic Clustering Protocol), HEED (Hybrid Energy-Efficient Distributed clustering), SFDCH (Secure and fault tolerant clustering for cluster head).

H.S. Annapurna et al. ${ }^{[4]}(\mathbf{2 0 1 5})$, In this paper, we propose a scheme called Secure Data Aggregation with Fault Tolerance for Wireless Sensor Networks which provides both end to end confidentiality and fault tolerance during data aggregation. Here, they propose to use shared cryptography to secure message communication in a sensor network. In this approach they divide the information into multiple shares and transmit the different shares via multiple disjoint paths between any pair of communicating nodes. At the receiving end the original information is reconstructed by combining the shares received via different paths.

Aby K. Thomas et al. ${ }^{[8]}(\mathbf{2 0 1 4})$, In this paper, they propose a re-clustering framework for wireless sensor networks to undergo global re-clustering and local delegation in order to enhance the lifetime of the network. The variation in energy distribution across the $\mathrm{CH}$ network can be characterized by a mapping function using a metric, based on energy cost, defined on a metric space. The mapping characterizes the change in link cost in terms of energy distribution. The change in link costs is defined as the distortion of the map. Distortion exceeding a given threshold is used to decide on re-clustering / local delegation.

Stefanos A. Nikolidakis et al. ${ }^{[10]}$ (2013), The lifetime of the sensor node is based on battery powered devices. Several authors discussed the different energy consumption techniques for different layers. Consolidated efficient energy consumption techniques are missing. This paper provides a detailed study about all the existing energy conservation techniques and also explains about limitations available in the techniques. In this paper, the energy conservation approaches and its algorithms for computing the optimal transmitting ranges in order to generate a network with desired properties are discussed.

Fan Xiangning et al. ${ }^{[25]}(\mathbf{2 0 0 7})$, This paper studies LEACH protocol, and puts forward energy-LEACH and multihop-LEACH protocols. Energy-LEACH protocol 
improves the choice method of the cluster head, makes some nodes which have more residual energy as cluster heads in next round. Multihop-LEACH protocol improves communication mode from single hop to multi-hop between cluster head and sink. Simulation results show that energy-LEACH and multihop-LEACH protocols have better performance than LEACH protocols.

\section{H-LEACH}

H-LEACH [1] being the combination of HEED and LEACH over comes the node energy issues, which is the major disadvantage of the LEACH protocol. H-LEACH is more efficient than existing LEACH protocol. H-LEACH uses residual and maximum energy of the nodes to elect a channel head for each round. An algorithm is used to find the life time of the nodes in terms of rounds when the threshold and energy conditions are considered. The nodes with energy less than to that of the (Etr) minimum energy required for transmitting and receiving signals is made to die as it lacks energy to do it. Etr is subtracted from the energy of the node s(i).e in every round as that much of energy is consumed. Total number of alive nodesare calculated for every round so as to have a track on the lifetime of the network.

\subsection{Working of H-LEACH [1]}

When the network enters the setup phase, Ep, the probability of using energy considerations is calculated by using Emax, $\mathrm{Cp}$ and Ep. Then the average energy of all the nodes are calculated. Then the threshold value is calculated. A number is randomly picked in the range 0 to 1. If the number picked is less than the threshold value and the corresponding node is assigned to be cluster head if its energy is more than that of the average energy. The energy required for data transmission is deduced from the energy of the node in every round. When the energy falls below the minimum value, it is declared to be dead. A graph is plotted for sum of alive nodes in each round. Evaluate $\mathrm{H}$ LEACH by apply Boltzmann Learning Boltzmann learning [3] is a stochastic recurrent neural network comprising of hidden layers and visible layers. The weights between the layers are adjusted according to the situation to give desired output .It is supervised learning and similar to error-correction learning where an error signal is used to train the system in each iteration. Each neuron in input layer is connected to hidden layer neurons and hidden layer neurons are connected to output layer neurons.

\section{Algorithm}

START ( )

1. Deploy sensor network with fixed number of sensor nodes

2. Apply location based clustering to cluster sensor nodes

3. Select cluster head in each cluster using H-LEACH protocol

4. If (link failure occurred in the network)
1. Apply Boltzmann learning to rate sensor nodes

2. Recover path through sensor nodes which has higher rating

Else

\{

Start communication from source to destination

\}

STOP

Results

Table of comparison

\begin{tabular}{|l|l|l|}
\hline Parameter & $\begin{array}{l}\text { Existing Algorithm } \\
\text { (H-LEACH) }\end{array}$ & $\begin{array}{l}\text { Proposed } \\
\text { Algorithm }\end{array}$ \\
\hline Delay & 240 packets & 180 packets \\
\hline $\begin{array}{l}\text { Energy } \\
\text { Consumption }\end{array}$ & 22 joules & 10 joules \\
\hline Packet-loss & 23 packets & 12 packets \\
\hline Throughput & 26 packets & 85 packets \\
\hline
\end{tabular}

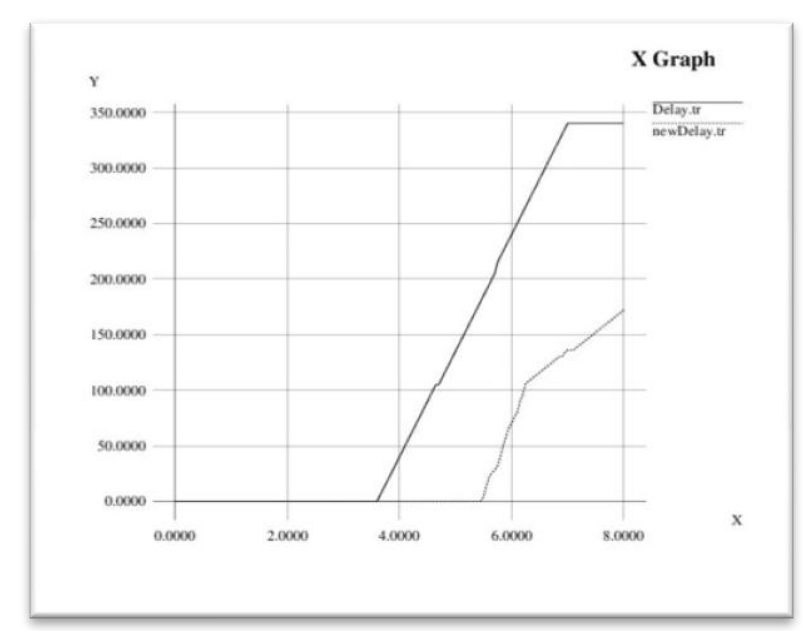

Delay Graph

As illustrated in graph, the delay of previous method and the delay observed in the new proposed work.

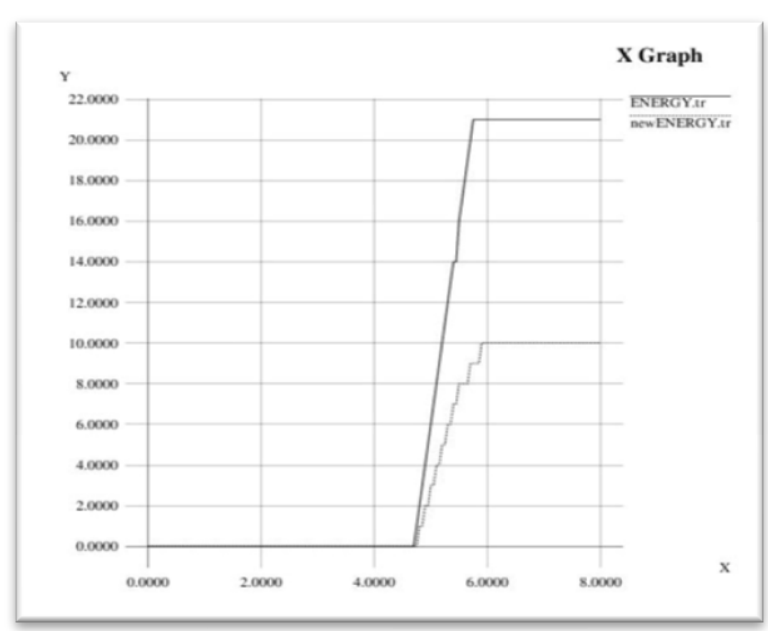

Energy Graph 
The performances are compared. The results show that the delay in the new proposed work is reduced by $30 \%$ due to fault removal in the network.

As shown in graph, the energy consumption of the $\mathrm{H}$ LEACH and new proposed work is compared. Due to the more fault in the network, the graph clearly shows that the energy consumption is more in the previous network. When fault is removed from the network, energy consumption is reduced from the network.

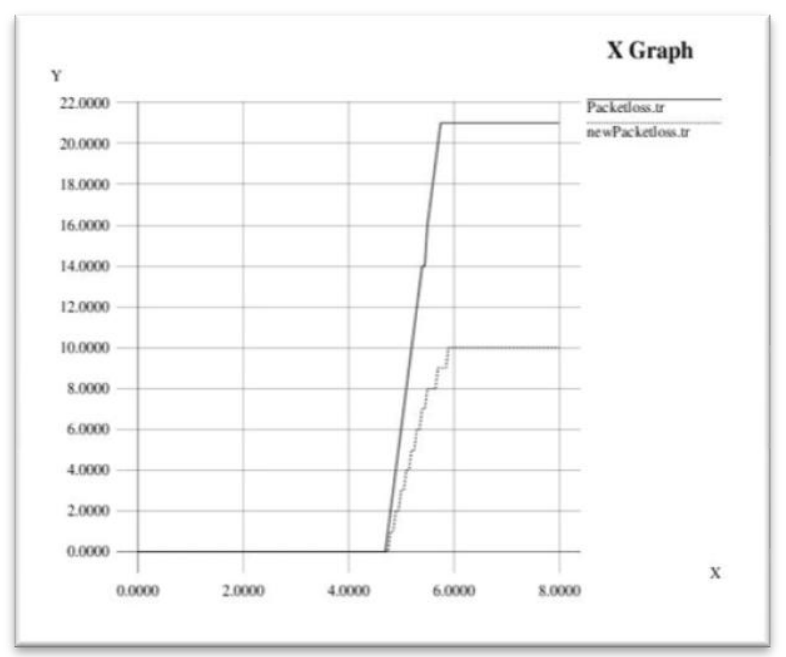

Packet loss Graph

As shown in graph, packet loss for the previous and new proposed work is compared. It is clearly seen in the graph that the packet loss in the previous work is more. It is due to the fault in the network and when this fault is removed from the network, the packet loss also gets reduced.

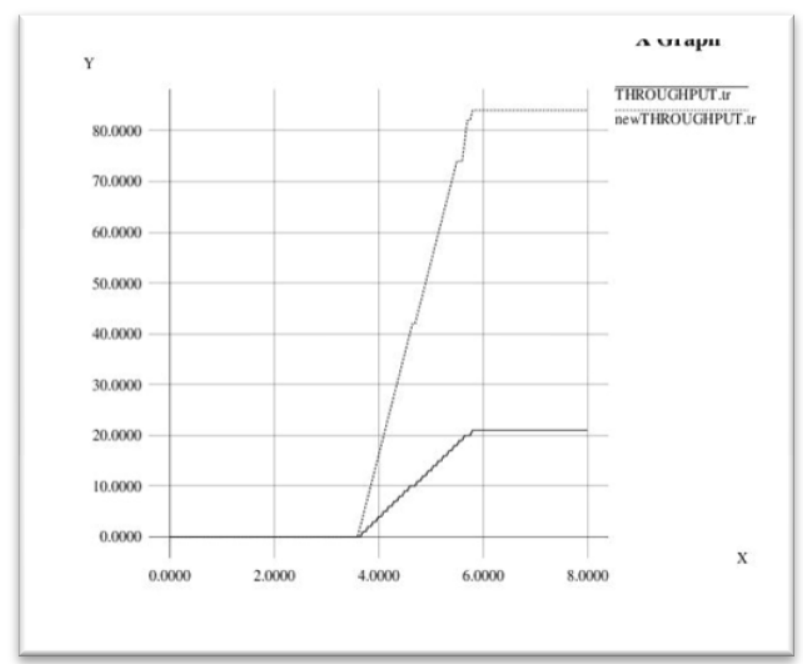

Throughput Graph

As shown in the graph, the throughput of the new and the previous work is compared. The throughput of the old scenario is reduced due to the fault in the network. If the fault of the network is recovered, the throughput of network increases accordingly.

\section{CONCLUSION}

In this paper, it is been concluded that various technique which reduced energy consumption of wireless sensor network is reviewed in terms of description and outcome. Due to small size of sensor nodes battery consumption is the major issue of wireless sensor networks. In this paper, we evaluate and analyze H-LEACH protocol by applying Boltzmann learning technique of neural networks to select intermediate node between Cluster Head and the Base Station for fault tolerance and to increase the network lifetime.

\section{REFERENCES}

[1] Abdul Razaque, Satwic Mudigulam, KiranGavini, Fathi Amsaad, Musbah Abdulgader, Gondal Sai Krishna (2016), " H-LEACH: Hybrid-Low Energy Adaptive Clustering Hierarchy for Wireless Sensor Networks". Long Island Systems, Applications and Technology Conference (LISAT), June 2016 IEEE.

[2] Blessy Varghese, "Cluster Head Selection Protocols in Wireless Sensor Networks". Imperial Journal of Interdisciplinary Research (IJIR) ISSN: 2454-1362, Vol-2, Issue-6, 2016

[3] Akshdeep Kaur, Ravneet Kaur (2016), "To introduce a fault tolerant multicasting protocol using boltzman neural network in WSN". International Journal of Engineering Development and Research Conference, Volume 4, Issue 3/ISSN: 2321-9939, 2016.

[4] H.S.Annapurna, M.Siddappa (2015), "Secure Data Aggregation with Fault Tolerance for Wireless Sensor Networks". International Conference on Emerging Research in Electronics, Computer Science and Technology 2015 IEEE.

[5] DavoodIzadi, Jemal Abawajy, Sara Ghanavati (2015), " An Alternative Clustering Scheme in WSN" 1530-437X (c) 2015 IEEE.

[6] Padmalaya Nayak and D. Anurag (2015), " A Fuzzy Logic based Clustering Algorithm for WSN to extend the Network Lifetime". IEEE Sensors Journal DOI 10.1109/JSEN.2015.2472970, IEEE 2015.

[7] Ajit R. Pagar, Prof. D. C. Mehetre (2015), "A Survey on Energy Efficient Sleep Scheduling in Wireless Sensor Network". International Journal of Advanced Research in Computer Science and Software Engineering,Volume 5, Issue 1, January 2015.

[8] Aby K. Thomas, R. Devanathan (2014)," Hybrid Re-Clustering Algorithm for Enhancement of Network Lifetime in Wireless Sensor Networks". International Review on Computers and Software (I.RE.CO.S.), Vol. 9, N. 9. September2014.

[9] Zhang, Degan, Guang Li, KeZheng, Xuechao Ming, and ZhaoHuaPan(2014), "An energy-balanced routing method based on forward-awarefactor for wireless sensor networks." Industrial Informatics, IEEETransactions on 10, no. 1 (2014): 766-773.

[10] Stefanos A. Nikolidakis, Dionisis Kandris, Dimitrios D. Vergados, Christos Douligeris,(2013) "Energy Efficient Routing in Wireless Sensor Networks Through Balanced Clustering". ISSN 1999-4893, Jan 2013.

[11] Arati Manjeshwar and Dharma P. Agrawa,(2002) "APTEEN: A Hybrid Protocol for Efficient Routing and Comprehensive Information Retrieval in Wireless Sensor Networks" At the Proceedings of the International Parallel and Distributed Processing Symposium, IEEE 2002.

[12] OssamaYounis, Sonia Fahmy,(2004) "HEED: A Hybrid, EnergyEfficient, Distributed Clustering Approach for Ad Hoc Sensor Networks" IEEE Transactions on Mobile Computing, VOL. 3, NO. 4, Oct-Dec 2004.

[13] Wendi Rabiner Heinzelman, Anantha Chandrakasan, and Hari Balakrishnan, (2000) "Energy-Efficient Communication Protocol for Wireless Micro sensor Networks" Proceedings of the 33rd Hawaii International Conference on System Sciences -IEEE 2000.

[14] Stephanie Lmdsey and Cauligi S. Raghavendra, (2001) "PEGASIS: Power-Efficient GAthering in Sensor Information Systems" IEEE 2002 . 
[15] Kunal M Pattani, Palak J Chauhan,(2015) "SPIN PROTOCOL FOR WIRELESS SENSOR NETWORK" International Journal of Advance Research in Engineering, Science \& Technology (IJAREST), Volume 2 ,Issue 5, May- 2015.

[16] Arati Manjeshwar and Dharma P. Agrawal, (2000) "TEEN: A Routing Protocol for Enhanced Efficiency in Wireless Sensor Networks" IEEE 2001.

[17] Shantala Devi Patil ,Vijaya kumar B P,(2016) "Overview of Issues and Challenges in Wireless Sensor Networks" International Journal of Application or Innovation in Engineering \& Management (IJAIEM) Volume 5, Issue 5, May 2016.

[18] S. Jerusha, K.Kulothungan, A. Kannan,(2012) "LOCATION AWARE CLUSTER BASED ROUTING IN WIRELESS SENSOR NETWORKS" International Journal of Computer \& Communication Technology ISSN (PRINT): 0975 - 7449, Volume3, Issue-5, 2012.

[19] Gagandeep Kaur, Kartik Sharma,(2016) "A Review on LEACH and its Descendants" International Journal of Computer Science and Mobile Applications, Vol.4 Issue. 5, May- 2016, pg. 21-28.

[20] D. Suresh and K. Selva kumar,(2015) "Double Cluster Head Based Reliable Data Aggregation for Wsn" World Engineering \& Applied Sciences Journal 6 (3): 136-146, 2015.

[21] P. J. Shriidhar1, Dr. A. Senthil Kumar,(2016) "A Collaborative Study on Selective, Energy Efficient and Clustering Based Hierarchical Routing Protocols for Wireless Sensor Networks" International Journal of Engineering Science and Computing, June 2016.

[22] Xiaodong Lin, Rongxing Lu and Xuemin (Sherman) Shen,(2009) "MDPA: multidimensional privacy-preserving aggregation scheme for wireless sensor networks" Wireless Communication \& Mobile. Computing (2009)

[23] Mohammad Shaifur Rahman, Youngil Park, and Ki-Doo Kim,(2009) "Localization of Wireless Sensor Network Using Artificial Neural Network" IEEE 2009.

[24] Mohammad Hossein Anisi, Javad Rezazadeh, Mehdi Dehghan, (2008) "FEDA: Fault-tolerant Energy-Efficient Data Aggregation in Wireless Sensor Networks" IEEE 2008.

[25] Fan Xiangning, Song Yulin, (2007) "Improvement on LEACH Protocol of Wireless Sensor Network "International Conference on Sensor Technologies and Applications, 2007.

[26] Sasanka Madiraju, Cariappa Mallanda, Rajgopal Kannan, Arjan Durresi, S.S.Iyengar,(2004) "EBRP: Energy Band based Routing Protocol for Wireless Sensor Networks" IEEE 2004. 